\title{
Evaluación del Modelo Climático Global MIROC5 y estimaciones de temperatura y precipitaciones para las zonas sur y norte del Perú
}

\section{MIROC5 Global Climate Model evaluation, temperature estimations and precipitations for Peru's southern and northern}

\author{
Aldo S. Moya Álvarez' \\ Universidad Continental \\ aldomoya00@gmail.com
}

\author{
Jose M. Ortega León ${ }^{2}$ \\ Centro Meteorológico de \\ Villa Clara, Cuba
}

\author{
Ximena Jurado Pacheco \\ Universidad Continental
}

\section{RESUMEN}

El objetivo radicó en evaluar los resultados del Modelo Climático Global MIROC5 para Perú y obtener una perspectiva del comportamiento futuro de las temperaturas y las precipitaciones para las zonas sur y norte del Perú. Se tomaron datos de salida "historical" del modelo, empleado por el IPCC en el Proyecto de Inter-comparación de Modelos Acoplados, fase cinco (CMIP5) y se realizó una verificación para el tiempo presente, a partir de la cual se ajustaron sus proyecciones simuladas y se obtuvieron las tendencias futuras de las precipitaciones y la temperatura hacia 2030, 2070 y 2100 . Como resultado se obtuvo un incremento de las precipitaciones hacia la zona norte del país $(27,6 \%$ hacia 2100$)$, que incluye la zona costera y la región de lquitos, al tiempo que puede esperarse una disminución hacia el sector sur. Para la temperatura se prevé un incremento en todo el país, pero las tendencias más pronunciadas se esperan en el sector sur del territorio. Hacia el 2030 para el escenario RCP 8.5 se estiman incrementos de hasta $1,16{ }^{\circ} \mathrm{C}$ para Pucallpa y $0,22{ }^{\circ} \mathrm{C}$ para Piura. Se concluye que las precipitaciones se incrementarán en el norte y disminuirán en el sur, mientras las temperaturas se incrementarán en todo el país, pero más significativamente en el sector sur.

Palabras clave: Cambio climático, modelos climáticos, escenarios climáticos, temperatura, precipitación.

\begin{abstract}
The objective was to evaluate the results of the MIROC5 Global Climate Model for Peru and to get a perspective of the temperatures future behavior and precipitations for the southern and northern sectors of the country. The "historical" output data from the model were taken, used by the IPCC in the Coupled Models Inter-comparison Project, stage 5 (CMIP5) and a verification was conducted for the present time, through this the simulated projections were adjusted and precipitation and temperature future trends towards 2030, 2070 and 2100 were obtained. As a result, a precipitation increase to the northern side of the country $(27,6 \%$ by 2100$)$ was obtained, It includes the coastal zone and the lquitos region, while a decrease can be expected to the south side. Temperature is expected to increase throughout the country, but the most pronounced trends are expected in the southern side of the territory. Towards 2030 RCP 8.5 scenario is expected to increase up to $1,16{ }^{\circ} \mathrm{C}$ for Pucallpa and $0,22{ }^{\circ} \mathrm{C}$ for Piura. So precipitations will increase in the northern and in the south will be lower, while temperatures will increase throughout the country, but more significantly in the southern side.
\end{abstract}

Keywords: Climate change, climate models, climate scenarios, temperature, precipitation.

Historial del artículo:

Recibido: 11 de octubre de 2015. Aprobado: 17 de noviembre de 2015. Disponible en línea: 30 de diciembre de 2015

1 Ingeniero Meteorólogo. Doctor en Ciencias Meteorológicas. Jefe de pronósticos de Villa Clara, Cuba. Investigador de la Universidad Continental, Perú.

2 Licenciado en Ciencias de la Computación, Centro Meteorológico de Villa Clara, Cuba

3 Estudiante de Ingeniería Ambiental de la Universidad Continental. 


\section{INTRODUCCIÓN}

El Cambio Climático es definido como un cambio estable y durable en la distribución de los patrones del clima en periodos de tiempo que van desde décadas hasta millones de años. Pudiera ser un cambio en las condiciones climáticas medias o en la distribución de eventos en torno a ellas, como serían, por ejemplo, los fenómenos meteorológicos extremos. En los últimos años el término "Cambio Climático" ha estado referido fundamentalmente al cambio causado por la actividad humana, a diferencia de aquellos causados por procesos naturales de la Tierra y el Sistema Solar. En este sentido, especialmente en el contexto de la política ambiental, el término "cambio climático" ha llegado a ser sinónimo de "calentamiento global antropogénico".

En 1988 la Organización Meteorológica Mundial (OMM) y el Programa de las Naciones Unidas para el Medio Ambiente (PNUMA) crearon el Grupo Intergubernamental de Expertos sobre Cambio Climático, más conocido por sus siglas en inglés como IPCC, con el objetivo de proporcionar información objetiva y clara sobre el estado de conocimientos acerca del cambio climático a los responsables políticos y otros sectores interesados.

Hasta el año 2014 el IPCC ha emitido cinco informes de evaluación del cambio climático, en los años 1990, 1995, 2001, 2007 y el más reciente entre los años 2013 y 2014. Para la realización de sus investigaciones el IPCC se basa fundamentalmente en los llamados Modelos Climáticos Globales (MCG), capaces de reproducir matemáticamente de una forma adecuada, los principales procesos que ocurren en los cinco componentes del sistema climático: atmósfera, océano, criosfera, geosfera y biosfera. Consisten en programas informáticos que se ejecutan en superordenadores con los que se resuelve numéricamente un conjunto de ecuaciones que expresan las leyes y principios de la física que gobiernan el sistema climático terrestre (1).

Perú es uno de los países de la llamada Comunidad Andina, cuyo clima es muy complejo por su diversidad, desde selvas tropicales hasta desiertos, pasando por la cordillera de los Andes. Los Andes han sido objeto de múltiples estudios sobre el tema del cambio climático, los cuales han abundado en sus perspectivas, impactos y consecuencias de diferentes maneras. Así, podemos encontrar en la literatura los trabajos de DeLucía et al. (2), Buytaert et al. (3), Caldas et al. (4), Hezog et al. (5), entre otros.

Para Perú existen varios precedentes relacionados con los escenarios de cambio climático hacia el futuro. Se encuentran entre ellos los trabajos de Marengo (6) y Marengo et al. (7) y Sanabria et al. (8), las Comunicaciones Nacionales de Cambio Climático $(9$ y 10). Igualmente se encuentran las investigaciones del Servicio Nacional de Meteorología e Hidrología (SENAMHI), sobre escenarios de Cambio Climático para Perú y para diferentes cuencas $(11,12,13,14)$.

En la literatura internacional se encuentran trabajos que intentan evaluar el funcionamiento de uno o varios modelos sobre una región determinada, lo cual suele hacerse utilizando, indistintamente, modelos globales o modelos regionales $(15,16)$. En otras investigaciones se acoplan modelos en forma de "Ensambles", con el objetivo de construir escenarios climáticos para un país o una región determinada. Se pueden señalar en este caso los trabajos de Cabazos et al (17).

En el presente trabajo se muestra algunos resultados para Perú a partir del modelo MIROC5, incorporado al proyecto CMIP5, que corresponde a la quinta fase del Proyecto de Intercomparación de Modelos Climáticos del Programa World Climate Research (WCRPs). El CMIP5 recoge un conjunto amplio de salidas modeladas que está disponible libremente a investigadores a través de un archivo de datos integrados y ha sido parte del quinto informe de evaluación del IPCC (18). El proyecto incluye simulaciones a largo plazo del clima del siglo XX y proyecciones para el siglo XXI y más allá. Recoge además, simulaciones a corto plazo centradas en las últimas décadas y el futuro hasta el año 2035. La principal particularidad de la investigación radica en que el modelo MIROC5 pertenece al grupo de modelos incorporados al CMIP5 y en que, el hecho de ajustar las simulaciones del modelo a partir de los resultados de la verificación, lo cual puede calificarse como una especie de reducción de escala estadística, permite obtener resultados más precisos en las perspectivas hacia los años 2030, 2070 y 2100.

\section{MATERIAL Y MÉTODOS}

El trabajo constituye una investigación aplicada. Para su realización se tomaron los datos de salida del modelo MIROC5, cuya procedencia y resolución se muestran en la tabla 1. Los escenarios tomados en cuenta fueron el RCP 8.5 y el RCP 4.5.

Los datos para validar el modelo fueron tomados de

Tabla № 1: Modelo MIROC5, empleado en la presente investigación (corrida run 1).

\begin{tabular}{lll}
\hline Modelo & \multicolumn{1}{c}{ Institución } & Resolución \\
\hline MIROC5 & National Institute $\mathrm{X}=1.406^{\circ}, \mathrm{Y}=1.39^{\circ}$ \\
& For Environmental \\
& Studies and Japan \\
& Agency for Marine- \\
& Earth Science and \\
& Technology, JAPÓN \\
\hline
\end{tabular}


siete estaciones meteorológicas de superficie del Perú, pertenecientes a la red del SENAMHI. La selección de las estaciones se hizo atendiendo a su ubicación geográfica, de manera que sus tendencias hacia el futuro brinden información sobre el posible clima del siglo XXI en diferentes sectores geográficos de la nación. Se tomaron los datos de temperatura media anual entre los años 1979 y 2000 . Para esta variable el estudio se concentró en las siete localidades, mientras que para las precipitaciones se emplearon solo cinco (se excluyeron Piura y Pucallpa), atendiendo a la disponibilidad de datos de las observaciones meteorológicas.

Para la temperatura se seleccionó el período antes mencionado atendiendo a que durante ese lapso se produjo el incremento más notable de esta variable en el siglo pasado, que se enmarcó entre mediados de la década de los años 70 y el año 2000, como se aprecia
De esta manera se puede comprobar que los modelos climáticos globales son capaces de reproducir bastante bien la distribución a gran escala de las principales variables climáticas y simulan de forma muy aceptable, los grandes rasgos del cambio climático observado. Gran parte de ellos coinciden cuantitativamente con muchos aspectos del cambio climático futuro (21).

No obstante, a escalas espaciales pequeñas se perciben algunas diferencias entre observaciones $y$ resultados, así como, discrepancias entre los propios modelos globales. Esto se atribuye, principalmente, a su baja resolución espacial, la cual trata de paliarse con la aplicación de modelos climáticos regionales más detallados o mediante el empleo de la llamada reducción de escala estadística, que intenta corregir las estimaciones de los modelos a partir de cómo hayan simulado el clima presente.

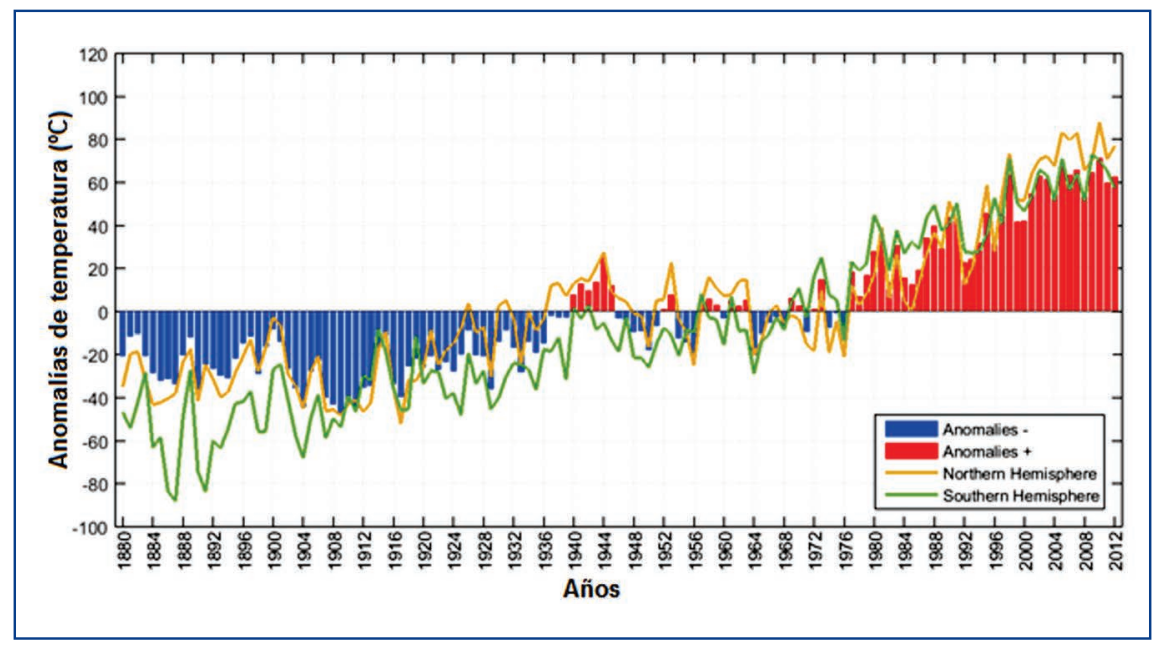

Figura $\mathrm{N}^{\circ} 1$ : Variación interanual de la temperatura media global y para los hemisferios sur y norte en el período 1880 - 2012 (Período base: 1951 - 1980).

en la figura 1 (19).

Para la verificación de las precipitaciones se emplearon las series de datos comprendidas entre los años 1984 y 1994 para las localidades de lquitos, Chiclayo y Cusco; entre 1986 y 1996 para la localidad de Arequipa y entre 1980 y 1990 para la localidad de Tacna, todas enmarcadas en el período 1979 al 2000, mencionado anteriormente. En este caso, el empleo de las series de datos de observaciones se realizó, además, atendiendo a la disponibilidad de datos suministrados por el SENAMHI.

La fiabilidad de los modelos climáticos se suele valorar analizando su destreza para reproducir el clima presente, su habilidad para simular los cambios climáticos observados en las últimas décadas o el grado de convergencia entre las proyecciones de cambio climático futuro que ofrecen (20).
Atendiendo a estos comentarios, se verificó el trabajo del modelo para el clima presente y las desviaciones resultantes fueron tomadas en cuenta para ajustar las simulaciones hacia el futuro, de forma tal, que los resultados obtenidos filtren los errores detectados durante la verificación.

La metodología se ejecutó de la siguiente forma:

1. Se descargaron los datos de temperaturas medias mensuales, de la salida del modelo y de las corridas "historical" del German Climate Computing Centre (22).

2. Se decodificaron los datos con ayuda del programa "DecodSC", desarrollado específicamente para decodificar dicha información.

3. Se realizó una interpolación para obtener los 
valores para cada punto, tanto de las salidas a futuro como de las corridas "historical". Para obtener los datos para cada punto seleccionado se empleó el método de interpolación "Spline Bicúbico". Este algoritmo sustituye a los viejos algoritmos de interpolación bicúbica por splines bicúbicos. Se trata de un algoritmo de interpolación de altas prestaciones, que proporciona resultados excelentes tanto en lo relativo a la velocidad de mencionadas. En este paso se emplearon dos métodos:

a) Se empleó la fórmula 1 de error medio absoluto ( $A M E$, por sus siglas en inglés), que permitió calcular la desviación media absoluta entre la serie simulada por cada modelo y la observada en cada estación meteorológica.

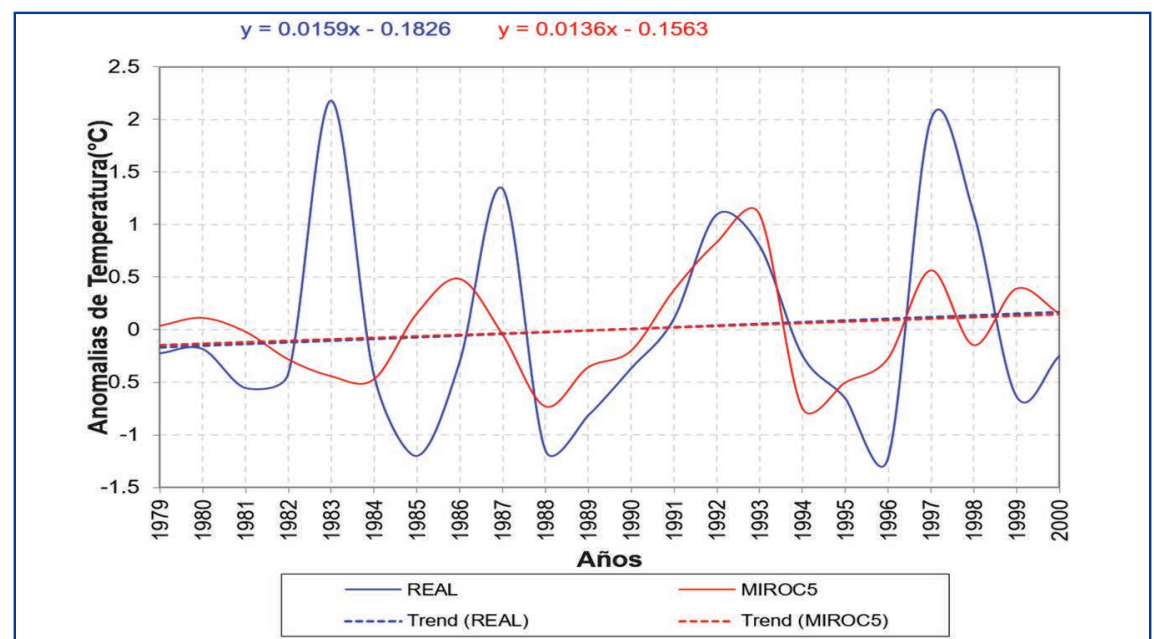

Figura $\mathrm{N}^{\circ}$ 2: Tendencias de la temperatura media anual observada y simulada por el modelo MIROC5 para el período 1979 - 2000 en Chiclayo.

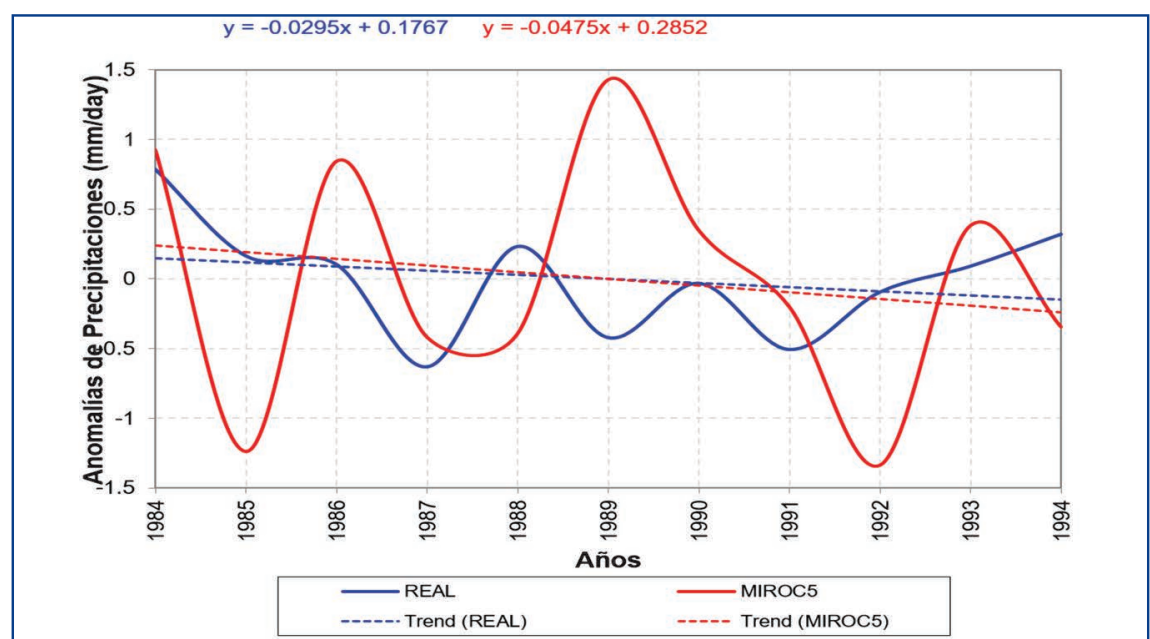

Figura $\mathrm{N}^{\circ}$ 3: Tendencias de la precipitación media anual observada y simulada por el modelo MIROC5 para el período 1984 - 1994 en Cusco.

ejecución como a la calidad de los resultados.

4. Se les realizó la prueba de homogeneidad de las medias anuales observadas a las siete estaciones meteorológicas tomadas para el estudio utilizando los estadísticos " $\mathrm{T}$ " de Student-Fisher $(\mathrm{P}<0,05)$.

5. Se realizaron las verificaciones para cada punto mediante el empleo de las corridas "historical" y los datos de las observaciones de las estaciones

$$
A M E_{a b s}=\frac{1}{N} \sum_{i}^{N}\left|\left[P_{F}(i)-P_{A}(i)\right]\right|
$$

Donde:

$A M E_{a b s}=$ Desviación media absoluta entre la variable observada y simulada, calculada para cada punto seleccionado. 
Tabla $\mathrm{N}^{\circ}$ 2: Tendencias $\left({ }^{\circ} \mathrm{C}\right)$ descritas por las simulaciones del modelo MIROC5 en las siete localidades estudiadas para el período 1979-2000.

\begin{tabular}{lc}
\hline \multirow{2}{*}{ Localidades } & Modelo Climático Global \\
\cline { 2 - 2 } Piura & MIROC5 \\
Chiclayo & 0,26 \\
lquitos & 0,28 \\
Cusco & 0,16 \\
Pucallpa & 0,36 \\
Arequipa & 0,91 \\
Tacna & 0,39 \\
Promedio & 0,46 \\
\hline
\end{tabular}

$P_{F}(i), P_{A}(i)=$ Variable, pronóstico y análisis respectivamente, para cada año de la serie tomada.

$N=$ Cantidad de años tomados para la verificación.

b) Se construyeron las curvas de comportamiento de las temperaturas simuladas y observadas entre los años 1979 y 2000, y se determinó su tendencia (se muestran como ejemplo las figuras 2 y 3). Seguidamente se determinaron los porcentajes de subestimación o sobreestimación del modelo en relación con las tendencias observadas a partir de los datos de las estaciones meteorológicas. Este paso permite tener una idea de en qué medida las tendencias descritas por el MIROC5 hacia el 2100 pueden estar siendo subestimados o sobrestimados.
Tabla № 3: Resultados de la verificación del funcionamiento del modelo MIROC5 respecto a las observaciones.

\begin{tabular}{llc}
\hline \multirow{2}{*}{ Localidades } & \multicolumn{2}{c}{ Modelo Climático Global } \\
\cline { 2 - 3 } & \multicolumn{2}{c}{ MIROC5 } \\
\cline { 2 - 3 } & AME & $\%$ T \\
\hline Piura & 0,69 & 450,0 \\
Chiclayo & 0,67 & $-14,0$ \\
Iquitos & 0,37 & 266,0 \\
Cusco & 0,50 & $-59,0$ \\
Pucallpa & 0,58 & 416,0 \\
Arequipa & 0,45 & $-45,0$ \\
Tacna & 0,37 & 159,0 \\
Promedio & 0,52 & 167,6 \\
\hline
\end{tabular}

6. Se construyeron las curvas de estimación desde el año 2006 hasta el 2100 para cada punto y escenario seleccionado. Se determinaron las tendencias y los cambios esperados hacia 2030, 2070 y 2100 , en relación con el período de referencia 1986 - 2005. Las tendencias fueron ajustadas a partir de los resultados obtenidos en el paso 5 .

\section{RESULTADOS}

Los resultados de la verificación del funcionamiento del modelo respecto a las observaciones para cada estación meteorológica, tomada en cuenta para la variable "temperatura media anual", se muestran en las tablas 2 y 3 . En la tabla 2 se presentan las tendencias descritas por las simulaciones del modelo en los siete departamentos y en la tabla 3 los resultados de la verificación con los datos de las observaciones. En esta última, "AME" es la desviación media absoluta entre

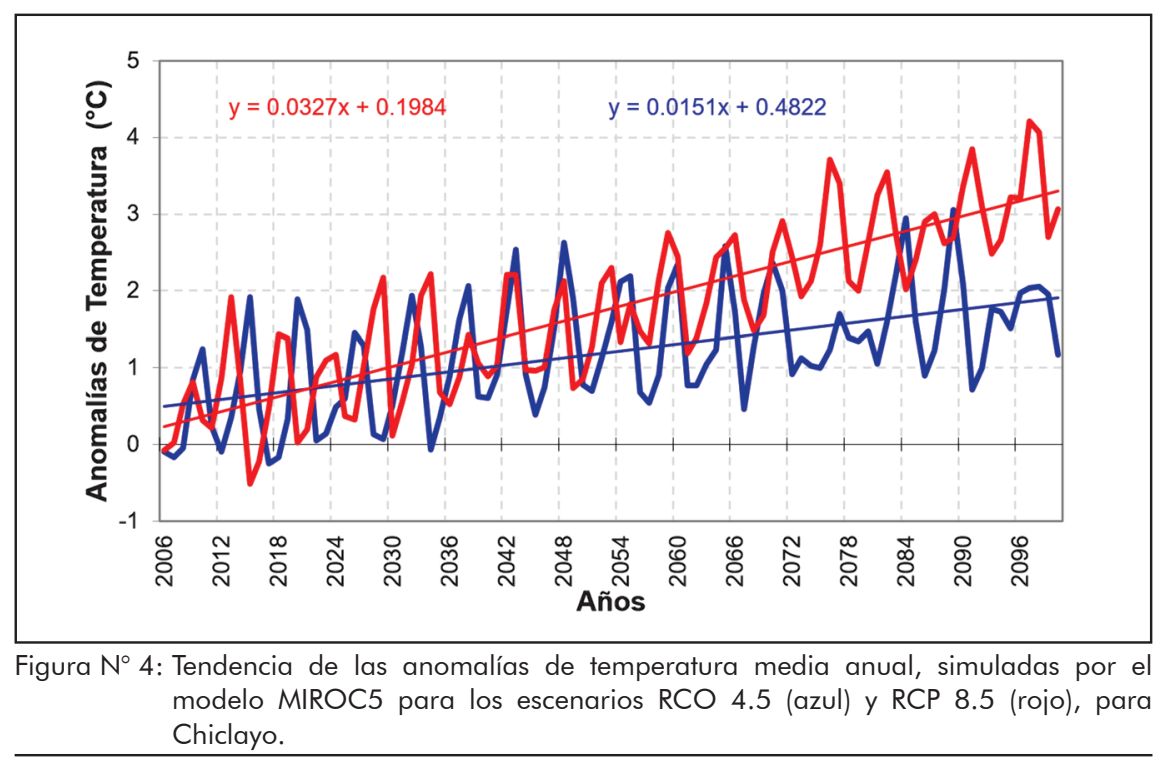


la temperatura media anual simulada y la observada en el período 1979 - 2000. El parámetro "\% T" es el porcentaje de sobreestimación o subestimación de las tendencias simuladas respecto a las observadas en dicho período. El signo negativo indica que el modelo subestimó la tendencia en relación con la observada.

En la tabla 2 se aprecia que en sentido general el modelo describió tendencias ascendentes de la temperatura.

En la tabla 3 se aprecia que de forma general el modelo sobrestimó la tendencia de la temperatura durante el período evaluado, como promedio, en un $167,6 \%$, anual hacia 2100 , así como su tendencia, simulada por el modelo MIROC5 para Chiclayo. De igual forma se construyeron para el resto de las ciudades.

En la tabla 4 se aprecia que los mayores incrementos se esperan en Pucallpa y Arequipa, mientras que en el norte los incrementos de temperatura estimados son menos significativos. En sentido general para el escenario RCP 8.5 se obtuvo el mayor incremento para Pucallpa con 1,$16 ; 3,41$ y $4,6{ }^{\circ} \mathrm{C}$; y los menores para Piura, con $0,22,0,52$ y $0,75^{\circ} \mathrm{C}$ para 2030,2070 y 2100 , respectivamente.

Para las precipitaciones se realizó un procedimiento

Tabla № 4: Anomalías esperadas $\left({ }^{\circ} \mathrm{C}\right)$ para los escenarios RCP 8.5 y RCP 4.5 hacia los años 2030, 2070 y 2100 para los cinco puntos seleccionados con referencia a la temperatura media anual del período 1986 - 2005.

\begin{tabular}{llcccccc}
\hline \multirow{2}{*}{ Localidades } & \multicolumn{3}{c}{ Anomalía RCP 8.5 } & \multicolumn{3}{c}{ Anomalía RCP 4.5 } \\
\cline { 2 - 8 } & 2030 & 2070 & 2100 & 2030 & 2070 & 2100 \\
\hline \multirow{2}{*}{ Norte } & Piura & 0,22 & 0,52 & 0,75 & 0,19 & 0,32 & 0,41 \\
& Chiclayo & 0,96 & 1,95 & 2,90 & 0,98 & 1,66 & 2,18 \\
& lquitos & 0,42 & 1,00 & 1,44 & 0,33 & 0,57 & 0,75 \\
& Pucallpa & 1,16 & 3,41 & 4,60 & 1,05 & 1,88 & 2,68 \\
Suryyyyyyyy & Cusco & 0,32 & 0,72 & 1,03 & 0,25 & 0,44 & 0,59 \\
& Arequipa & 1,07 & 3,36 & 4,56 & 1,01 & 1,76 & 2,14 \\
& Tacna & 0,75 & 1,83 & 2,64 & 0,63 & 1,13 & 1,50 \\
\hline
\end{tabular}

Tabla $N^{\circ}$ 5: Tendencias ( $\left.\mathrm{mm} / \mathrm{día}\right)$ descritas por las simulaciones del modelo MIROC5 en las siete ciudades para el período de 11 años estudiado.

\begin{tabular}{cc}
\hline \multirow{2}{*}{ Localidades } & Modelo Climático Global \\
\cline { 2 - 2 } & MIROC5 \\
\hline Chiclayo & 0,86 \\
Iquitos & $-0,20$ \\
Cusco & $-0,48$ \\
Arequipa & 0,03 \\
Tacna & 0,13 \\
General & 0,45 \\
\hline
\end{tabular}

con los valores más elevados para Piura, lquitos y Pucallpa. Las mejores simulaciones tuvieron lugar para Arequipa y Chiclayo. En el caso de la desviación media absoluta, esta resultó de $0,52{ }^{\circ} \mathrm{C}$, de forma general, con el valor más bajo para lquitos y Tacna.

\section{Simulaciones a futuro}

Sobre la base de estos resultados se construyeron las curvas de tendencia para cinco ciudades y los escenarios RCP 8.5 y RCP 4.5.

La figura 4 muestra la curva de comportamiento esperado de las anomalías de temperatura media
Tabla № 6: Resultados de la verificación del funcionamiento de los cinco modelos seleccionados respecto a las observaciones.

\begin{tabular}{lrr}
\hline \multirow{2}{*}{ Localidades } & \multicolumn{2}{c}{ Modelo Climático Global } \\
\cline { 2 - 3 } & \multicolumn{2}{c}{ MIROC5 } \\
\cline { 2 - 3 } & AME & \% T \\
\hline Chiclayo & 0,37 & 2664 \\
lquitos & 0,75 & -111 \\
Cusco & 0,36 & 61 \\
Arequipa & 0,46 & 120 \\
Tacna & 0,32 & 2740 \\
General & 0,45 & 1090 \\
\hline
\end{tabular}

similar. La tabla 5 muestra las tendencias descritas por el modelo para cada ciudad estudiada en los períodos correspondientes (clima presente). En general el modelo estimó incremento de las precipitaciones para tres de las cinco ciudades.

En la tabla 6 se representan los resultados obtenidos para los parámetros "AME" $\mathrm{y}$ "\% T". Los resultados dejan claro que el modelo sobrestimó significativamente en sus pronósticos con excepción de lquitos. El mejor pronóstico se realizó para Cusco.

La figura 5 representa la curva de comportamiento esperado de las anomalías de precipitación media 


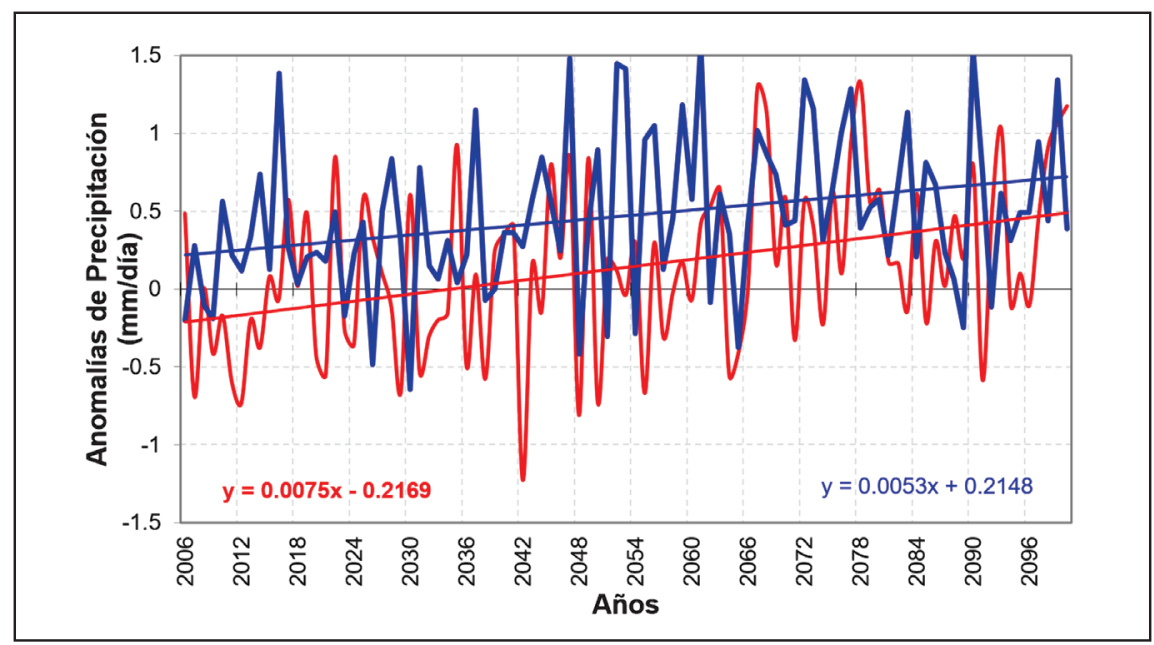

Figura $\mathrm{N}^{\circ}$ 5: Tendencia de las anomalías de la precipitación media anual, simuladas por el modelo MIROC5 para los escenarios RCP 4.5 (azul) y RCP 8.5 (rojo), para lquitos.

Tabla № 7: Anomalías esperadas (\%) para los escenarios RCP 8.5 y RCP 4.5 hacia los años 2030, 2070 y 2100 , para los cinco puntos seleccionados con referencia a la precipitación media anual ( $\mathrm{mm} /$ días) del período 1986 - 2005.

\begin{tabular}{llcccccc}
\hline \multirow{2}{*}{ Localidades } & \multicolumn{3}{c}{ Anomalía RCP 8.5 } & \multicolumn{3}{c}{ Anomalía RCP 4.5 } \\
\cline { 2 - 8 } & 2030 & 2070 & 2100 & 2030 & 2070 & 2100 \\
\hline \multirow{2}{*}{ Norte } & Chiclayo & 0,6 & 1,6 & 2,4 & 0,79 & 1,22 & 1,55 \\
& lquitos & 8,3 & 19,1 & 27,6 & 12,4 & 20,1 & 25,6 \\
\multirow{2}{*}{ Sur } & Cusco & $-8,4$ & $-3,7$ & $-0,3$ & $-9,3$ & $-8,0$ & $-7,0$ \\
& Arequipa & $-0,6$ & $-1,8$ & $-6,7$ & $-1,9$ & $-1,8$ & $-4,2$ \\
& Tacna & $-0,0$ & $-0,0$ & $-0,0$ & $-0,0$ & $-0,0$ & $-0,0$ \\
\hline
\end{tabular}

anual hacia 2100, así como su tendencia, simulada por el modelo para lquitos.

En la tabla 7 se muestran los resultados obtenidos para cada ciudad. El modelo sugiere un decrecimiento en el régimen de precipitaciones para las localidades del sur (Cusco, Arequipa y Tacna) y un incremento para las localidades en el norte, ligeramente hacia la ciudad de Chiclayo y de forma más marcada hacia la zona selvática de lquitos.

El signo negativo en los valores $-0,0$, para Tacna, se debe a que las tendencias esperadas son descendentes, pero sus módulos se enmarcan en el orden de las centésimas.

\section{DISCUSIÓN}

Los resultados obtenidos coinciden con otros estudios realizados sobre el tema, aunque con datos pertenecientes a experimentos anteriores del IPCC, en que los mayores incrementos de temperatura deben esperarse hacia el centro y sur del país. Rosas et al (13) publicaron que los incrementos de temperatura a esperar en la zona alta de Piura (norte de Perú) estarían entre 0,2 y $2{ }^{\circ} \mathrm{C}$ hacia 2030 , mientras que para el sur estimó incrementos superiores a los $2{ }^{\circ} \mathrm{C}$ para el mismo período. De manera que los resultados obtenidos en el presente trabajo coinciden en que los mayores incrementos estarían en el sur del país disminuyendo hacia el norte, pero con incrementos menos significativos en ambos casos.

Para el caso de las precipitaciones los resultados coinciden con los publicados en la segunda comunicación nacional (10), que señaló incrementos de las precipitaciones hacia la costa norte y en la zona de lquitos, con una disminución en la zona de Tacna, extremo sur del país. En este caso, el crecimiento en la zona de lquitos es significativo. Hacia el sur se espera una disminución de las precipitaciones en relación con el periodo 1986 - 2005.

En conclusión, para el escenario RCP 8.5 se esperan los mayores incrementos de temperaturas hacia las ciudades de Pucallpa, Arequipa y Tacna (entre 0,7 y $1,2{ }^{\circ} \mathrm{C}$ hacia 2030), al igual que para la ciudad costera de Chiclayo (norte). Los incrementos más discretos se obtuvieron para las ciudades de Piura, Cusco e lquitos (entre 0,2 y $0,5^{\circ} \mathrm{C}$ hacia 2030). De manera general, los incrementos más notables se esperan hacia el centro y sur del país. Se sugieren incrementos de las precipitaciones hacia la costa norte (Chiclayo) y en la 
selva nororiental del país, mientras en las ciudades de Cusco, Tacna y Arequipa se espera precipitaciones inferiores a las registradas en el período 1986 - 2005. Para el escenario RCP 4,5 los resultados son similares, aunque en el caso de la temperatura, los incrementos a esperar son más discretos.

\section{Agradecimientos}

Al Servicio Nacional de Meteorología e Hidrología del Perú (SENAMHI) por facilitar el acceso a los datos meteorológicos del periodo 1979 - 2000; asimismo a la Universidad Continental por los recursos financieros para la ejecución de este trabajo de investigación.

\section{REFERENCIAS BIBLIOGRÁFICAS}

1. McGuffie K, Henderson-Sellers A. A Climate Modelling Primer. 3rd ed. Wiley J, Sons, editors. Chichester: Wiley; 2005.

2. DeLucia EH, Casteel CL, Nabity PD, O'Neill BF. Insects take a bigger bite out of plants in a warmer, higher carbon dioxide world. Proc Natl Acad Sci U S A. 2008; 105(6): p. 1781-1782.

3. Buytaert W, Célleri R, Timbe L. Predicting climate change impacts on water resources in the tropical Andes: Effects of GCM uncertainty. Geophysical Research Letters. 2009; 36(7): p. 1-11.

4. Zapata-Caldas E, Jarvis A, Ramirez J, Lau C. Potenciales impactos del Cambio Climático en cultivos andinos. Serie Panorama Andino sobre Cambio Climático Lima-Quito: CONDESANSGCAN; 2011.

5. Herzog SK, Martínez R, Jørgensen PM, Tiessen H. Climate change and biodiversity in the Tropical Andes. Paris: Inter-American Institute for Global Change Research (IAI) and Scientific Committee on Problems of the Environment (SCOPE); 2011.

6. Marengo JA. Escenarios de Cambio Climático em América del Sur: Impactos, vulnerabilidad y adaptación em relación a extremos. 2007. Disponible en: http://pnud.cptec.inpe.br/itens/ entrenamiento2/apresentacoes/Escenarios\%20 de $\% 20$ cambio $\% 20$ climatico $\% 20$ en $\% 20$ America\%20del\%20Sur_Jose\%20Marengo.pdf.

7. Marengo JA, Jones R, Alves LM, Valverde MC. Future change of temperature and precipitation extremes in South America as derived from the PRECIS regional climate modeling system. International Journal of Climatology. 2009; 29(15): p. 2241-2255.

8. Sanabria J, Marengo JA, Valverde M. Escenarios de Cambio Climático com modelos regionales sobre el Altiplano Peruano (departamento de Puno). Revista Peruana Geo-Atmosférica. 2009;(1): p. 134-149.
9. Ministerio del Ambiente del Perú. Primera Comunicación Nacional del Perú a la Convención de Naciones Unidas sobre Cambio Climático. Informe técnico. Lima: MINAM, Consejo Nacional del Ambiente; 2001.

10. Ministerio del Ambiente del Perú. Segunda Comunicación Nacional del Perú a la Convención de Naciones Unidas sobre Cambio Climático. Informe técnico. Lima: MINAM, Consejo Nacional del Ambiente; 2010.

11. Servicio Nacional de Meteorología e Hidrología. Escenarios de cambio climático en la cuenca del río Mantaro para el año 2100. Informe técnico. Lima: SENAMHI, Centro de Predicción Numérica; 2007.

12. Servicio Nacional de Meteorología e Hidrología. Escenarios de cambio climático en la cuenca del río Urubamba para el año 2100. Informe técnico. Lima: SENAMHI, Centro de Predicción Numérica; 2009.

13. Servicio Nacional de Meteorología e Hidrología. Escenarios climáticos en el Perú para el año 2030. Informe técnico. Lima: SENAMHI, Centro de Predicción Numérica; 2009.

14. Servicio Nacional de Meteorología e Hidrología. Escenarios climáticos en la cuenca del río Santa para el año 2030. Informe técnico. Lima: SENAMHI, Centro de Predicción Numérica ; 2009.

15. Blázquez J. Proyecciones climáticas sobre Sudamérica utilizando modelos climáticos globales. Análisis de incertidumbres. Tesis doctoral. Buenos Aires: Universidad de Buenos Aires, Facultad de Ciencias Exactas y Naturales; 2011.

16. Alcafuz R. Simulaciones climáticas para Chile entre los años 2030 y 2060. In Taller Regional de Metrología y Retos Tecnológicos en las Ciencias del Clima y la Energía Renovable; 2014; Montevideo.

17. Cavazos T, Salinas JA, Martínez B, Colorado C, Grau P, et al. Actualización de escenarios de cambio climático para México como parte de los productos de la Quinta Comunicación Nacional. Informe técnico. México D.F.: Instituto Nacional de Ecología y Cambio Climático; 2013.

18. Taylor E, Stouffer J, Meehl A. An Overview of CMIP5 and the Experiment Design. Bull. Amer. Meteor. Soc. 2012; 93(4): p. 485-498.

19. NASA. Goddard Institute for Space Studies. [Online].; 2015 [cited 2014 Noviembre 15]. Available from: http://www.giss.nasa.gov/.

20. Raïsänen J. How reliable are climate models? Tellus. 2007; 59(1): p. 2-29.

21. AchutaRao K, Covey C, Doutriaux C, Fiorino M, Gleckler P, et al. An Appraisal of Coupled Climate Model Simulations Bader D, editor. California: Lawrence Livermore National Laboratory; 2004.

22. Deutsches Klimarechenzentrum. [Online]. [cited 2014 Noviembre 14]. Available from: http://www. dkrz.de/daten-en/cera/data_base. 